\title{
CXIV. OBSERVATIONS ON ACETYLCHOLINE.
}

\author{
BY HAROLD WARD DUDLEY. \\ From the National Institute for Medical Research, Hampstead, London.
}

(Received August 22nd, 1929.)

IN a recent investigation of the physiologically active constituents of an alcoholic extract of horse's spleen [Dale and Dudley, 1929] acetylcholine was, for the first time, isolated and identified as a constituent of an animal tissue.

Chemical observations, incidental to this work, were made, which seem worthy of separate record for the guidance of any who may be faced with the problem of the isolation of this compound from natural sources.

There are two earlier reports of its identification as a natural product; in each case the source was vegetable, namely, ergot [Ewins, 1914], and shepherd's purse (Capsella bursa pastoris) [Boruttau and Cappenberg, 1921].

The method of isolation described by Ewins was, in the main, followed by Boruttau and Cappenberg and, with more extensive modification, in our own work on spleen extract. In all cases the penultimate product was a mixture of chloroplatinates, precipitated from alcoholic solution.

Choline has properties so similar to those of its acetyl derivative that it inevitably accompanies the latter through the preliminary purifications, and, being widely distributed in animal and vegetable tissues, it formed the chief constituent in the mixture of chloroplatinates obtained in the three investigations mentioned above. In each case, therefore, the final stage of the isolation presented the difficult problem of separating a small proportion of acetylcholine chloroplatinate from a large proportion of choline chloroplatinate.

Ewins, relying on the statement in the literature that the chloroplatinate of acetylcholine is less soluble than that of choline, attempted to increase this disparity by fractional crystallisation of his mixture from water at $35^{\circ}$ and obtained a less soluble chloroplatinate forming orange-coloured polyhedra and melting at $256-7^{\circ}$. His analysis of the salt showed a slight excess of platinum $(28.4 \%$; theoretical $27 \cdot 8 \%$ ).

Since, however, after acetylating choline according to Nothnagel's directions [1894] he obtained a chloroplatinate identical in chemical properties and physiological activity with that derived from ergot, he concluded that the latter was acetylcholine chloroplatinate, although Nothnagel describes the salt as crystallising in bright yellow needles, melting at $223-4^{\circ}$.

The mixture of chloroplatinates obtained in our work from $32 \mathrm{~kg}$. of horse's spleen weighed $2.7 \mathrm{~g}$. and the physiological activity of the chlorides 
liberated from a sample of the mixture corresponded to only about onetwentieth of this weight of acetylcholine chloroplatinate. Before an attempt was made to separate the latter from so great an excess of impurity, chiefly choline chloroplatinate, it was necessary to investigate the relative solubilities of the two chloroplatinates; for, unless the difference was indeed great, the possibility of obtaining a chemically pure specimen of the acetylcholine salt appeared to be remote, particularly as recrystallisation from hot water was known to cause partial hydrolysis of this substance. In view of eventual identification and on account of the disagreement between Nothnagel's and Ewins's descriptions, it was also important to examine the properties of pure acetylcholine chloroplatinate itself.

Choline was, accordingly, acetylated by heating it at $100^{\circ}$ with acetyl chloride [Nothnagel, 1894] and the chloroplatinate of acetylcholine was found to crystallise in strongly anisotropic needles, melting at $242^{\circ}$, agreeing, except as to melting-point, with Nothnagel's description.

In the numerous acetylations which were carried out, variable amounts of another chloroplatinate were frequently encountered. It crystallised in isotropic octahedra of an orange-yellow colour and had a melting-point corresponding to that given by Ewins. This substance, on account of its much smaller solubility in water, could be easily separated from the anisotropic needles which always formed the main product of the acetylation.

The anisotropic needles had the correct platinum content of acetylcholine chloroplatinate, whilst the regular octahedra contained more platinum than the formula of this compound required. Moreover, the chloride derived from this octahedral salt by decomposition with potassium chloride had only $53 \%$ of the physiological activity of that similarly prepared from the anisotropic needles.

Analysis having proved that the salt crystallising in needles was pure acetylcholine chloroplatinate, the nature of the octahedral material was investigated.

It was eventually found to be a double salt, containing one molecule of choline chloroplatinate and one of acetylcholine chloroplatinate; i.e. cholineacetylcholine dichloroplatinate. This complex salt is much less soluble than acetylcholine chloroplatinate, which in its turn is less soluble than choline chloroplatinate. It is precipitated when appropriate aqueous solutions of choline and acetylcholine chloroplatinates are mixed, and crystallises from water in isotropic orange-yellow octahedra melting at $260-1^{\circ}$. That it is a definite chemical compound was proved by mixing solutions of choline and acetylcholine chloroplatinates in molecular proportions of $1: 3,1: 1$ and $3: 1$; in each experiment the double salt, containing one molecule of each component, separated. The observation that, contrary to the usual behaviour of mixed melting-point determinations, a mixture of choline chloroplatinate (M.P. $240^{\circ}$ ) and acetylcholine chloroplatinate (M.P. $242^{\circ}$ ) melted at a higher temperature $\left(248^{\circ}\right)$ than did either of the components, furnishes additional evidence of compound formation by the two salts. 
Choline-acetylcholine dichloroplatinate, in all probability, owesits existence to co-ordination between the hydrogen atom of the hydroxyl group of the choline moiety and the oxygen atom of the acetyl group in acetylcholine. If this is so it may suggest a structural basis for the well-known dimorphism exhibited by choline chloroplatinate, which crystallises from water in anisotropic prismatic needles and from $50 \%$ alcohol in isotropic polyhedra. It seems reasonable to assume that the latter form may actually be a co-ordination compound, consisting of two molecules of choline chloroplatinate, produced by the co-ordination of the hydrogen atoms of the two hydroxyl groups of one molecule with the oxygen atoms of the two hydroxyl groups of the second molecule. According to this view the anisotropic salt, crystallised from water, is choline chloroplatinate, whilst the isotropic salt, crystallised from $50 \%$ alcohol, is dicholine dichloroplatinate. This conception is supported by the fact that acetylcholine chloroplatinate, in which there is no similar hydrogen atom available for co-ordination, crystallises in anisotropic needles both from water and from alcohol of all strengths up to $70 \%$ and, in this investigation, has never exhibited dimorphism.

With the elucidation of the nature and properties of choline-acetylcholine dichloroplatinate various observations made during this study became intelligible. It had been found, for instance, that a solution of pure acetylcholine chloroplatinate in ten volumes of water, kept at $37^{\circ}$ for some days, slowly deposited the octahedral salt. The explanation of this phenomenon is that acetylcholine salts are gradually hydrolysed in aqueous solution and consequently the choline chloroplatinate, so produced, combined with the unchanged acetylcholine salt to form the relatively insoluble dichloroplatinate, until, when half of the acetylcholine had been hydrolysed, the solution contained only choline-acetylcholine dichloroplatinate. On heating an aqueous solution of this double salt at $100^{\circ}$ for six hours, hydrolysis of the acetylcholine component continued and pure choline chloroplatinate only was recovered.

The occurrence of variable amounts of the octahedral salt in the chloroplatinate isolated after acetylating choline also became explicable: any choline, either escaping acetylation or arising from the readily hydrolysable acetyl derivative during the process of isolation, combined with a molecular equivalent of acetylcholine to produce the dichloroplatinate.

It is now obvious that from any mixture of the chloroplatinates of choline and acetylcholine, on crystallisation from water, choline-acetylcholine dichloroplatinate will be formed in amount equivalent to that of whichever component is present in smaller quantity. Only after the latter has been removed as the double salt will it be possible to obtain from the mother-liquor the remainder of the simple chloroplatinate of the component which is present in excess, and it will never be possible to isolate the simple chloroplatinate of the less abundant component.

A fact of practical importance is that the solubility of the dichloroplatinate is considerably depressed in the presence of excess of choline chloroplatinate; 
to such an extent, indeed, that on mixing appropriate solutions containing one part of acetylcholine chloroplatinate and nineteen parts of choline chloroplatinate respectively, the acetylcholine was recovered practically quantitatively as choline-acetylcholine dichloroplatinate.

These observations have an important bearing on the isolation of acetylcholine from natural sources. In the three cases already mentioned the crude chloroplatinates contained a small quantity of the acetylcholine salt and a large excess of that of choline, and it is reasonable to assume that similar proportions would be obtained in any other fractionation of biological material which might contain acetylcholine. On recrystallising such a mixture from water, therefore, all the acetylcholine would inevitably appear as cholineacetylcholine dichloroplatinate. It was this salt which was obtained in our investigation of spleen; and it was this salt which Ewins secured in his research on ergot, although, by the isolation of the same compound (for reasons explained above) from the reaction-mixture obtained on acetylating choline, he was misled as to its true composition. It is clear that the present investigation in no wise invalidates his work; it offers, indeed, complete confirmation of the fact that he obtained acetylcholine from ergot, but shows that he separated it as choline-acetylcholine dichloroplatinate.

Boruttau and Cappenberg [1921], instead of crystallising their crude chloroplatinate from water as did Ewins, extracted it with warm $70 \%$ alcohol in which they assert acetylcholine chloroplatinate to be fairly soluble and choline chloroplatinate practically insoluble. This statement was tested but not confirmed. Both salts were found to be soluble to the extent of approximately $0.5 \%$ in $70 \%$ alcohol at $37^{\circ}$, and, in agreement with this determination, an extraction of a mixture of the two salts with this solvent failed to remove preferentially that of acetylcholine. Their method therefore is ineffective and, accordingly, did not lead to a satisfactory isolation of acetylcholine chloroplatinate. The chemical and physiological properties of the active substance which they investigated, however, make it very probable that they were in fact handling acetylcholine. Their gravimetric estimate of the amount of acetylcholine present in extracts of shepherd's purse is surprisingly high and must be grossly inaccurate, since the salt they weighed for this purpose must have been choline-acetylcholine dichloroplatinate and not, as they thought, acetylcholine chloroplatinate, quite apart from the probability of the weighed fraction containing other relatively insoluble chloroplatinates (e.g. those of ammonium and potassium).

It will be clear from the foregoing discussion that the formation of the double chloroplatinate of choline and acetylcholine, and particularly its relative insolubility in the presence of excess of the choline salt, makes the isolation of acetylcholine from natural sources much more simple than would otherwise be the case.

As a matter of interest an experiment was made to ascertain whether the chloroaurates of choline and acetylcholine would yield a co-ordination com- 
pound similar to that of the chloroplatinates, but no such compound was obtained on mixing equimolecular equivalents of the two salts. The experiment led, however, to the useful observation that recrystallisation of such a mixture from water easily yielded, with comparatively little loss, pure acetylcholine chloroaurate, which is much less soluble than the choline salt. By liberating the chlorides, therefore, from choline-acetylcholine dichloroplatinate, converting them into chloroaurates and recrystallising the mixture from water, it proved to be a simple matter to obtain a pure specimen of acetylcholine chloroaurate. This process was successfully applied to the dichloroplatinate isolated from horse's spleen, thus providing a further chemical identification of the acetylcholine which this organ contained.

For the isolation of acetylcholine from the reaction-mixture obtained after acetylating choline the chloroaurate is much superior to the chloroplatinate. The former salt is very considerably less soluble in water than the latter and, as no relatively insoluble co-ordination compound is produced, the presence of unacetylated choline introduces no complication. Further, whilst choline and acetylcholine chloroplatinates melt (with decomposition) at $240^{\circ}$ and $242^{\circ}$ respectively, the corresponding chloroaurates melt at $266^{\circ}$ (with decomposition) and $166^{\circ}$ (without decomposition); a better chemical criterion of purity is therefore available for acetylcholine chloroaurate.

When acetylcholine chloroaurate is decomposed by hydrogen sulphide, hydrolysis occurs simultaneously, choline chloride being produced [Nothnagel, 1894]. It is this fact which hitherto has made the chloroplatinate the only practicable metallic salt from which acetylcholine chloride could be liberated for physiological experiment; for in this case decomposition with hydrogen sulphide can be avoided, and the chloride of the base can be obtained by evaporating to dryness a solution of the chloroplatinate, to which potassium chloride has been added, and extracting the residue with alcohol, in which acetylcholine chloride is soluble.

The chloroaurate has, however, been made available as a source of acetylcholine chloride by the discovery of a most convenient and rapid method of decomposition in which no concomitant hydrolysis occurs. It consists simply in shaking an aqueous solution of the salt with excess of metallic silver. Thereby, in a few minutes, the gold and its associated chlorine atoms are precipitated as metal and silver chloride respectively; these, together with the excess of silver, are removed by filtration, a neutral aqueous solution of the chloride of the base being obtained. The method is equally applicable to other chloroaurates and also to chloroplatinates. In addition to its speed and simplicity it has the advantage that no free hydrochloric acid is formed, as happens when hydrogen sulphide is used. It should, therefore, prove useful in general practice, particularly with salts of easily decomposable bases. The reactions may be formulated as follows:

$$
\begin{aligned}
& \mathrm{RAuCl}_{4}+3 \mathrm{Ag}=\mathrm{Au}+3 \mathrm{AgCl}+\mathrm{RCl} \\
& \mathrm{R}_{2} \mathrm{PtCl}_{6}+4 \mathrm{Ag}=\mathrm{Pt}+4 \mathrm{AgCl}+2 \mathrm{RCl} .
\end{aligned}
$$


It should be noted that the chlorine (or hydrochloric acid) associated with basic nitrogen is not removed in the reaction, even in the presence of excess of silver.

\section{EXPERIMENTAL.}

\section{Acetylcholine chloroplatinate.}

Dry choline chloride is heated with ten times its weight of acetyl chloride in a sealed tube at $100^{\circ}$ for 3 hours [see Nothnagel, 1894]. The excess of acetyl chloride is then removed in vacuo, in a water-bath at $30^{\circ}$. The syrupy residue is dissolved in a small quantity of absolute alcohol. After standing for 10 minutes to decompose any remaining acetyl chloride, the solution is again evaporated under the same conditions. The residue is taken up in alcohol and precipitated by the addition of alcoholic platinum chloride solution. The crude chloroplatinate is dissolved in 5 volumes of hot water. If cholineacetylcholine dichloroplatinate is present in appreciable quantity it separates in octahedral form as the solution cools, acetylcholine chloroplatinate crystallising later in light yellow needles. The solution is allowed to stand for some hours and is then cautiously warmed until the needles are just redissolved. It is then filtered quickly through a warm funnel, leaving behind undissolved octahedra. From the cooled filtrate are obtained the needles, which may still be contaminated with a small quantity of octahedra. For final purification the former are dissolved in 10 volumes of water at $50^{\circ}$ and allowed to crystallise at about $5^{\circ}$. The process is repeated, if necessary, until homogeneous needles are obtained. As these are anisotropic and the contaminating octahedra isotropic, a polarising microscope is useful in following the purification. Acetylcholine chloroplatinate forms thin, bright yellow needles; M.P. $242-4^{\circ}$ (with decomposition). It crystallises well from $50 \%$ alcohol.

Analysis. $0.1047 \mathrm{~g}$. gave $0.0292 \mathrm{~g}$. Pt.

Found: $\mathrm{Pt}, 27.9 \%$.

Calc. for $\mathrm{C}_{14} \mathrm{H}_{32} \mathrm{O}_{4} \mathrm{~N}_{2} \mathrm{Cl}_{6} \mathrm{Pt}$ : Pt, $27 \cdot 8 \%$.

It may be noted in passing that Baeyer, who first prepared acetylcholine [1867], acetylated choline in the cold and Nothnagel was unable to repeat this experiment. Baeyer's observation was, however, confirmed during the present investigation by an experiment in which a solution of choline chloride in acetyl chloride was allowed to stand overnight: $70 \%$ of the choline was acetylated.

\section{Choline-acetylcholine dichloroplatinate.}

$1 \mathrm{~g}$. choline chloroplatinate was dissolved in $10 \mathrm{cc}$. water and $1 \mathrm{~g}$. acetylcholine chloroplatinate in $12 \mathrm{cc}$. water at $45^{\circ}$. On mixing the two solutions a sandy precipitate was at once formed. After standing, the precipitate was filtered off and weighed $1 \cdot 1 \mathrm{~g}$. This was recrystallised from $10 \mathrm{cc}$. water, $0.75 \mathrm{~g}$. orange-yellow isotropic octahedra being obtained; M.P. $260-1^{\circ}$ (with decomposition). 
Analysis. $0 \cdot 2058 \mathrm{~g}$. gave $0 \cdot 0610 \mathrm{~g}$. Pt.

Found: Pt, $29 \cdot 6 \%$.

Calc. for $\mathrm{C}_{24} \mathrm{H}_{60} \mathrm{O}_{6} \mathrm{~N}_{4} \mathrm{Cl}_{12} \mathrm{Pt}_{2}$ : $\mathrm{Pt}, 29 \cdot 6 \%$.

The mother-liquor from the preparation, on slow evaporation, deposited the remainder of the compound in large well-formed octahedra.

Evidence for constancy of composition. The following aqueous solutions were mixed:

acetylcholine chloroplatinate choline chloroplatinate

(a) $0.2 \mathrm{~g}$. (approx. $1 \mathrm{~mol}$ ) in $2.5 \mathrm{cc}$. (at $\left.45^{\circ}\right)+0 \cdot 2 \mathrm{~g}$. (approx. $1 \mathrm{~mol}$ ) in $2 \mathrm{cc}$.

(b) $0.2 \mathrm{~g}$. ( $1 \mathrm{~mol}$ ) in $2.5 \mathrm{cc}$. (at $\left.45^{\circ}\right)+0.528 \mathrm{~g}$. $(3 \mathrm{mols})$ in $2 \mathrm{cc}$.

(c) $0.6 \mathrm{~g}$. (3 mols) in $5 \mathrm{cc}$. (at $\left.45^{\circ}\right)+0.176 \mathrm{~g}$. $(1 \mathrm{~mol})$ in $1 \mathrm{cc}$.

In each experiment an immediate sandy precipitate was produced. After standing overnight, these were collected and weighed: $(a) 0.215 \mathrm{~g} .,(b) 0.324 \mathrm{~g}$., (c) $0.247 \mathrm{~g}$.

On recrystallisation from 10 volumes of water each crop yielded identical octahedra, melting with decomposition at $260-1^{\circ}$, and containing the correct platinum content of the double salt. The physiological activities of the chlorides liberated from the three specimens were the same and $53 \%$ of that of an equal weight of the acetylcholine salt. The physiological activity of acetylcholine is about a thousand times greater than that of choline; the latter, therefore, makes no appreciable contribution to the activity of a solution containing the two substances in equimolecular proportions. As the acetylcholine component comprises $53 \%$ of the weight of choline-acetylcholine dichloroplatinate, the activity of the chlorides derived from it corresponded with the theoretical anticipation.

Solubility relationships. The yields of the double salt obtained in the three experiments illustrate the lowering of its solubility in the presence of excess of either component. Even in Exp. (c), where the total volume of the solution was 6 cc. as compared with $4.5 \mathrm{cc}$. in $(a)$ and $(b)$, and in which the solution had to be warmed to $37^{\circ}$, before filtration, to dissolve the excess of acetylcholine chloroplatinate which had crystallised out, the yield was, nevertheless, greater than in (a).

This lowering of solubility is more strikingly illustrated by the following experiment where the conditions are similar to those encountered in the fractionation of spleen extract. $2.85 \mathrm{~g}$. choline chloroplatinate were dissolved in $7 \mathrm{cc}$. water; the salt remained in solution at $37^{\circ}$ but crystallised on cooling to room temperature. $0 \cdot 15 \mathrm{~g}$. acetylcholine chloroplatinate, dissolved in $1 \mathrm{cc}$. water, was mixed with the solution of the choline salt at $37^{\circ}$, when a fine precipitate formed. The mixture was heated on the water-bath until a clear solution resulted; the hot liquid after standing at $37^{\circ}$ for 3 hours was filtered, $0 \cdot 281 \mathrm{~g}$. choline-acetylcholine dichloroplatinate being collected, which, after one recrystallisation, was pure.

It will be noted that the recovery of the added acetylcholine chloroplatinate 
in the form of the double salt was practically quantitative; obviously even smaller proportions of acetylcholine could be successfully isolated from similar mixtures.

\section{Acetylcholine chloroaurate.}

Choline is acetylated as already described. After removing the excess of acetyl chloride the residue is dissolved in water and immediately precipitated with excess of aqueous gold chloride solution. The chloroaurate is recrystallised from 30 volumes of water, the hot solution being filtered, if necessary, to remove the small amount of metallic gold which may be produced.

Acetylcholine chloroaurate crystallises in irregular thin glistening plates; M.P. $166-8^{\circ}$. It crystallises well from $1 \%$ acetic acid.

Analysis. 0.1175 g. gave 0.0478 g. Au.

Found: $\mathrm{Au}, 40 \cdot 7 \%$.

Calc. for $\mathrm{C}_{7} \mathrm{H}_{16} \mathrm{O}_{2} \mathrm{NCl}_{4} \mathrm{Au}: \mathrm{Au}, 40 \cdot 6 \%$.

The ease with which acetylcholine chloroaurate can be separated from an equimolecular equivalent of the choline salt is illustrated by the following experiment. Hot aqueous solutions of $0.22 \mathrm{~g}$. choline chloroaurate $(4 \mathrm{cc}$.) and $0.24 \mathrm{~g}$. acetylcholine chloroaurate $(6 \mathrm{cc}$.) were mixed. The material which crystallised on standing weighed $0.315 \mathrm{~g}$. and melted at $154-6^{\circ}$. After one recrystallisation from $10 \mathrm{cc}$. water $0.206 \mathrm{~g}$. acetylcholine chloroaurate (M.P. $166-8^{\circ}$ ) was obtained.

Preparation of acetylcholine chloroaurate from choline-acetylcholine dichloroplatinate isolated from spleen extract. The dichloroplatinate $(0 \cdot 2 \mathrm{~g}$.) from horse's spleen [Dale and Dudley, 1929] was dissolved in $10 \mathrm{cc}$. water; the solution was shaken with $0.4 \mathrm{~g}$. silver (see following section), filtered when the reaction was complete and then treated with $10 \%$ aqueous gold chloride solution. After standing for 12 hours at $3^{\circ}$ the precipitated chloroaurate was filtered off and weighed 0.0848 g.; M.P. $152-4^{\circ}$. It was recrystallised from 2.5 cc. water, $0.0689 \mathrm{~g}$. of a chloroaurate indistinguishable from that of acetylcholine being obtained. Simultaneous determination of the melting-points of this chloroaurate, pure acetylcholine chloroaurate and a mixture of the two resulted in all three specimens melting sharply at $166-8^{\circ}$.

Analysis. $0 \cdot 03160 \mathrm{~g}$. gave $0 \cdot 01280 \mathrm{~g}$. Au.

Found: $\mathrm{Au}, 40.5 \%$.

Calc. for $\mathrm{C}_{7} \mathrm{H}_{16} \mathrm{O}_{2} \mathrm{NCl}_{4} \mathrm{Au}: \mathrm{Au}, 40 \cdot 6 \%$.

The salt is therefore identified as acetylcholine chloroaurate.

New method for the decomposition of chloroaurates and chloroplatinates.

Metallic silver is prepared by mixing equal volumes of a $20 \%$ solution of ferrous sulphate crystals in $0.2 N$ sulphuric acid and a $4 \%$ silver nitrate solution in water. The finely divided metal so obtained is washed with water until free from sulphate, dried, and kept in a well-stoppered bottle. 
The salt to be decomposed is dissolved in water, twice its weight of silver is added and the mixture is shaken vigorously for a few minutes. When decomposition is complete, the excess silver, silver chloride and precipitated metal are removed by filtration and a solution of the chloride (or hydrochloride) of the base is obtained.

Application to the salts of acetylcholine. In order to ascertain whether acetylcholine chloride could be liberated by this method from the chloroaurate and chloroplatinate without removal of the acetyl group by hydrolysis, the following experiment was performed.

To a solution of $1.1 \mathrm{mg}$. acetylcholine chloroplatinate in 2 cc. water was added $0.1 \mathrm{cc} .5 \% \mathrm{KCl}$ solution. The mixture was evaporated to dryness over sulphuric acid in vacuo. The residue was extracted with 5 cc. absolute alcohol four times. After removal of the alcohol in vacuo at $35^{\circ}$ from the filtered extract, the residue was dissolved in 10 cc. water. This solution $(S)$ served as the standard of comparison for the physiological testing of solutions prepared by decomposing the chloroplatinate $(1.45 \mathrm{mg}$.) and chloroaurate $(3.4 \mathrm{mg}$.) of acetylcholine by the silver method. In each case the salt was dissolved in about 5 cc. water and shaken for 2-3 minutes with excess of silver in a stoppered measuring cylinder. The solution, being then colourless, was made up to 20 cc. and filtered. The solutions ( $P=$ that from the chloroplatinate; $A=$ that from the chloroaurate) were tested against the standard by comparing their actions on a strip of rabbit's isolated intestine [Dale, 1914]. For the actual test $S$ and $A$ were diluted 25 times and $P 12.5$ times. Equality of action was observed between 0.35 cc. $S(1 / 25)$ and 0.28 cc. $P(1 / 12.5)$ and between 0.25 cc. $S(1 / 25)$ and 0.22 cc. $A(1 / 25)$.

From these results the physiological determination of the amount of acetylcholine chloride in $P$ is $94 \%$ and in $A 102 \%$ of that calculated theoretically. These estimates are within the limits of accuracy of the method.

It is clear, therefore, that the silver method liberates acetylcholine chloride without hydrolysis from both the chloroplatinate and the chloroaurate.

Application to spermine chloroaurate. In order to ascertain whether the salts of primary and secondary amines behaved similarly to those of quaternary ammonium bases, the chloroaurate of spermine was chosen for experiment, since this base contains two amino- and two imino-groups [Dudley, Rosenheim and Starling, 1926].

$0 \cdot 1$ g. spermine chloroaurate was dissolved in $20 \mathrm{cc}$. water on a water-bath: while the solution was still warm, $0 \cdot 2 \mathrm{~g}$. silver was added and the mixture was briskly shaken. The solution became colourless in about 1 minute and, after filtration and evaporation, yielded the crystalline hydrochloride. This was converted into the picrate, which crystallised in needles and melted with decomposition at $248-50^{\circ}$, as did authentic spermine picrate and a mixture of the two specimens.

The method is, therefore, applicable to the salts of primary and secondary amines. 


\section{Test of Boruttau and Cappenberg's method for separating choline and acetylcholine chloroplatinates.}

$0.558 \mathrm{~g}$. choline-acetylcholine dichloroplatinate was converted into chlorides by shaking its solution in $15 \mathrm{cc}$. water with $1 \mathrm{~g}$. silver. The filtered solution was taken to dryness in vacuo, first by distillation to small volume at $35^{\circ}$ and finally over sulphuric acid in a desiccator. The residue was dissolved in absolute alcohol and precipitated with alcoholic platinum chloride solution. The chloroplatinate $(0.516 \mathrm{~g}$.) was digested with two successive quantities of $25 \mathrm{cc}$. $70 \%$ alcohol at $35^{\circ}$ for periods of 2 hours. Under these conditions $0 \cdot 1461 \mathrm{~g}$. chloroplatinate was recovered from the evaporated extracts in the form of isotropic octahedra (choline-acetylcholine dichloroplatinate).

A physiological test on small samples of the unextracted salt, the extracted substance and the residue indicated that all three materials had practically identical activities and that, therefore, $70 \%$ alcohol had not removed acetylcholine chloroplatinate preferentially. Since in this experiment the chloroplatinates of choline and acetylcholine were present in equimolecular proportions, the conditions were more favourable for the extraction of the acetylcholine salt than would be the case in material from biological sources.

Solubilities of choline and acetylcholine chloroplatinates in $70 \%$ alcohol. The two salts, in excess, were warmed in $70 \%$ alcohol to $50^{\circ}$ and then kept at $37^{\circ}$ for 6 hours with frequent shaking. 4 cc. of each of the solutions, after filtration, were evaporated to dryness in vacuo over sulphuric acid and the residues weighed: $0.0194 \mathrm{~g}$. choline chloroplatinate and 0.0203 g. acetylcholine chloroplatinate were obtained. The acetylcholine salt is therefore only very slightly more soluble in $70 \%$ alcohol than that of choline.

\section{Summary.}

1. The properties of the chloroplatinate and chloroaurate of acetylcholine have been studied.

2. Acetylcholine chloroplatinate, which crystallises in anisotropic needles, forms a co-ordination compound with one molecule of choline chloroplatinate. This double salt, choline-acetylcholine dichloroplatinate, is less soluble than either of its components and crystallises in isotropic octahedra.

3. A structural basis for the well-known dimorphism exhibited by choline chloroplatinate is suggested.

4. The significance of choline-acetylcholine dichloroplatinate in connection with the isolation of acetylcholine from natural sources is discussed.

5. A further identification of acetylcholine, isolated from horse's spleen as choline-acetylcholine dichloroplatinate, is provided by the preparation of acetylcholine chloroaurate from the double chloroplatinate.

6. A simple method, of general application and advantage, for the preparation of chlorides (or hydrochlorides) of bases from their chloroplatinates and chloroaurates is described. It consists in shaking aqueous solutions of these 
salts with metallic silver. Its special usefulness in connection with relatively unstable bases is illustrated by the fact that, when applied to acetylcholine chloroaurate, acetylcholine chloride is liberated quantitatively, whereas the alternative treatment with hydrogen sulphide results in concomitant hydrolysis with the formation of choline chloride.

I wish to thank Dr J. H. Gaddum and Mr L. W. Collison for making many physiological determinations of acetylcholine during the course of this investigation.

\section{REFERENCES.}

Baeyer (1867). Liebig's Ann. 142, 322.

Boruttau and Cappenberg (1921). Arch. Pharm. 259, 33.

Dale (1914). J. Pharm. Exp. Ther. 6, 147.

— and Dudley (1929). J. Physiol. 68, 97.

Dudley, Rosenheim and Starling (1926). Biochem. J. 20, 1082.

Ewins (1914). Biochem. J. 8, 44.

Nothnagel (1894). Arch. Pharm. 232, 266. 